\title{
MODELING DME INTERFERENCE IMPACT ON LDACS1
}

\author{
Ulrich Epple, Felix Hoffmann, and Michael Schnell \\ German Aerospace Center (DLR), Wessling, Germany
}

\begin{abstract}
LDACS1 is a candidate for the future digital aeronautical communications system in L-band. As unused spectrum is very scarce in the L-band, LDACS1 pursues the approach to make use of the gaps between adjacent channels used by the distance measuring equipment (DME). This DME signal has a severe influence on the performance of LDACS1. In this paper, an algorithm for modeling the DME impact on LDACS1 is presented. This enables to determine whether LDACS1 can cope with DME interference for a certain area and channel frequency. The performance evaluation is based only on the positions, pulse rates, and transmit frequencies of the DME stations, without carrying out extensive simulation. Results for Europe indicate that a reliable operation of LDACS1 can be achieved.
\end{abstract}

\section{Introduction}

The L-band digital aeronautical communications system (LDACS) is the air-to-ground data link technology within the future communications infrastructure (FCI). Jointly developed by Eurocontrol and the Federal Aviation Administration (FAA), the FCI comprises current and future communications technologies, which are required to implement the modernization of air-traffic management (ATM) as currently pursued within SESAR [1] and NextGen [2] in Europe and the US, respectively.

Two candidate systems for LDACS have been selected from a rich variety of proposals and the ICAO recommended to further investigate both systems in detail. LDACS1 is the broadband candidate technology for LDACS and employs orthogonal frequency-division multiplexing (OFDM) as modulation. It is designed as a frequency-division duplex system. LDACS2 is a narrowband singlecarrier system utilizing time-division as duplex scheme. In the remainder of this paper, we concentrate on LDACS1.

LDACS 1 is intended to be operated in the aeronautical part of the L-band (960-1164 MHz). The frequency band is already utilized by different legacy systems. This includes aeronautical navigation aids such as the distance measuring equipment (DME) or the military tactical air navigation (TACAN) system as well as communication systems like the military joint tactical information distribution system (JTIDS). In addition, several fixed channels are allocated for surveillance and collision avoidance; the universal access transceiver (UAT) at $978 \mathrm{MHz}$, the secondary surveillance radar (SSR) and traffic collision avoidance system (TCAS) at 1030/1090 MHz.

Due to these systems, free spectral resources are scarce and difficult to allocate in the aeronautical part of the L-band. For providing a sufficiently high capacity for current and future ATM applications, LDACS1 pursues the approach to make use of the gaps between adjacent DME channels. This inlay deployment of LDACS1 is the preferred deployment approach. However, the system design also allows for alternatively deploying LDACS1 without inlay in unused parts of the L-band if made available.

In this paper, we investigate the impact of DME interference, which is the main L-band interference source, on LDACS1 for the inlay deployment scenario. We concentrate on the LDACS1 forward link (FL) transmission from ground stations (GS) to airborne stations (AS) where interference is caused only by DME stations on ground. Compared to the reverse link (RL) the FL is seen as the more critical case and in addition less complex to model. The main goal of this investigation is to develop an appropriate model which describes the impact of DME interference on LDACS1 without requiring timeconsuming simulations. The resulting model uses an interference map of Europe, which describes DME interference by its main parameters: pulse rate, power distribution, and transmit frequency. Using these parameters, the model converts DME interference into an equivalent signal-to-noise ratio (SNR) for an LDACS 1 transmission. Based on this model we are able to determine if constraints for the LDACS1 deployment in Europe have to be taken into account. These constraints might be valid either globally or 
only for hotspots of DME interference in Europe. Constraints for the deployment comprise the necessity for frequency planning or a restriction on the maximum cell size. Also information if the foreseen interference mitigation techniques are sufficient could be deduced.

The remainder of the paper is organized as follows. After a brief overview of the key LDASC1 physical layer parameters, we characterize the DME system. Next, we present algorithms for mitigating the DME interference at the LDACS1 receiver side. The main part of the paper covers the modeling of the DME impact onto LDACS1. That comprises a DME interference map for Europe and the derivation of a model, which allows us to transform the interference map into an equivalent SNR loss of the LDACS1 transmission. Having confirmed the validity of the derived model, we further investigate the influence of the DME interference onto LDACS1 based on the model. In particular we assess under which conditions the LDACS1 performance criterion can be fulfilled. In the last section, conclusions are drawn and a short outlook for further work is given.

\section{LDACS1 System Overview}

In this section, we give a brief overview of the LDACS1 system, where special focus is put on the physical layer, since the physical received signal is directly affected by DME signals. For more detailed information please refer to the LDACS1 system specification [3], [4].

\section{Spectral Deployment}

LDACS1 is intended to operate in the lower part of the L-band (960-1164 MHz). It is designed as a frequency-division duplex system, which enables a GS to transmit continuously at a certain frequency, while the AS transmit at the same time but at a different frequency. For LDACS1, the frequency spacing between FL and the RL is set to $63 \mathrm{MHz}$.

For the LDACS1 deployment in the L-band different scenarios are possible. The most challenging approach is the inlay scenario where the LDACS1 channels with a bandwidth of approximately $500 \mathrm{kHz}$ are placed in between the existing DME channel grid of $1 \mathrm{MHz}$ with an offset of $500 \mathrm{kHz}$ to the DME center frequencies, as explained in the next section. This approach allows an LDACS1 deployment without changing existing DME assignments. For the inlay scenario the frequency range from 985.5 to $1008.5 \mathrm{MHz}$ is foreseen for the FL whereas the RL should be placed in the frequency range from 1048.5 to $1071.5 \mathrm{MHz}$. This spectral deployment minimizes the mutual interference between LDACS1 and other L-band systems, mainly SSR Mode S and UAT and is illustrated in Figure 1.

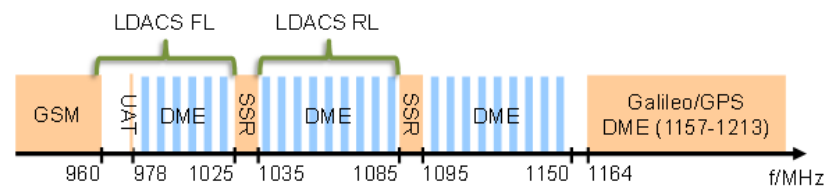

Figure 1. Spectral Deployment of LDACS1

\section{Physical Layer Design}

The LDACS1 signal is a multi-carrier signal, based on OFDM technology. The FL is a continuous OFDM transmission while the RL is based on orthogonal frequency-division multiple-access/ timedivision multiple-access (OFDMA-TDMA) bursts assigned to different users on demand. This enables aircraft to adopt their duty-cycle and the allocation of different subcarriers according to the interference conditions. Another feature which is integrated into LDACS1 to adapt the throughput to the interference conditions is adaptive coding and modulation (ACM), where different coding rates and modulation schemes are supported. For strong interference, the most robust setting is quadrature phase shift keying (QPSK) modulation and a concatenated coding scheme with a rate 0.5 convolutional code (CC) and a rate 0.9 Reed-Solomon (RS) code.

The channel bandwidth of $498.05 \mathrm{kHz}$ is used by an OFDM system with 50 subcarriers, resulting in a subcarrier spacing of $9.77 \mathrm{kHz}$. This subcarrier spacing was chosen as a trade-off between a high spectral efficiency and an acceptable inter-carrier interference (ICI) caused by Doppler spreads typically occurring in the aeronautical environment.

For OFDM modulation, a 64-point FFT is used. The total FFT bandwidth comprising all subcarriers is $625.0 \mathrm{kHz}$. Besides the 50 subcarriers used for transmission, the 64 subcarriers comprise a direct current (DC) subcarrier as well as seven empty subcarriers at the left edge of the spectrum and six at the right edge, serving as guard bands. In addition, pilot subcarriers are inserted for transmission channel estimation at the receiver. Exemplary, the structure of 
an FL OFMD symbol in the frequency domain is depicted in Figure 2.

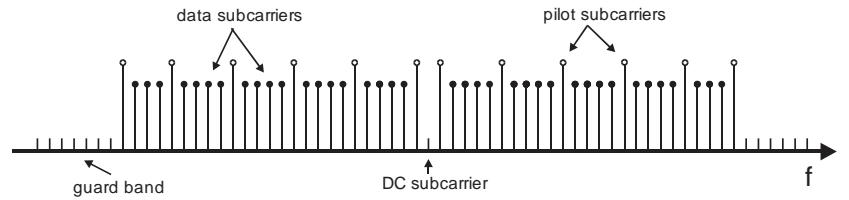

Figure 2. Structure of LDACS1 OFDM Symbol in Frequency Domain

According to the subcarrier spacing, one OFDM symbol has a duration of $102.4 \mu \mathrm{s}$. Each OFDM symbol is extended by a cyclic prefix of $17.6 \mu \mathrm{s}$, comprising a guard interval of $4.8 \mu \mathrm{s}$ as well as $12.8 \mu \mathrm{s}$ for transmit windowing. The guard interval provides resistance to inter-symbol interference caused by multipath effects. Transmit windowing leads to a reduction of the out-of-band radiation. This results in a total OFDM symbol duration of $120 \mu$ s. The main LDACS1 OFDM parameters are listed in Table 1.

Table 1. LDACS1 OFDM Parameters

\begin{tabular}{|l|l|}
\hline Parameter & Value \\
\hline Effective bandwidth (FL or RL) & $498.05 \mathrm{kHz}$ \\
\hline Subcarrier spacing & $9.765625 \mathrm{kHz}$ \\
\hline Used subcarriers & 50 \\
\hline FFT length & 64 \\
\hline OFDM symbol duration & $102.4 \mu \mathrm{s}$ \\
\hline Cyclic prefix & $17.6 \mu \mathrm{s}$ \\
$-\quad$ guard time & $4.8 \mu \mathrm{s}$ \\
- windowing time & $12.8 \mu \mathrm{s}$ \\
\hline Total OFDM symbol duration & $120 \mu \mathrm{s}$ \\
\hline
\end{tabular}

\section{Framing Structure}

OFDM symbols are organized into LDACS1 frames. Depending on their functionality, different frame types are distinguished. The frames are arranged into multi-frames (MF) and super-frames (SF). The structure of a SF is depicted in Figure 3.

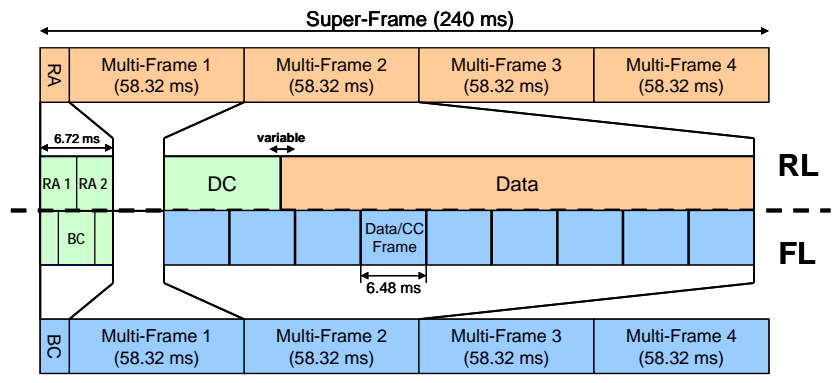

Figure 3. Super-Frame Structure

One SF comprises one broadcast (BC) frame in the FL and one random access (RA) frame in the RL, respectively, and four MF in both, FL and RL. Each MF itself contains nine data/CC frames in the FL and one dedicated control (DC) segment and one data segment in the RL.

\section{DME System Overview}

In [5], it was concluded that DME signals represent the most severe interference towards LDACS1. In this section we describe the signal shape of the DME pulses and its spectrum. In addition we explain the working principle of the DME system. This allows us to derive values for typical and worstcase squitter rates.

\section{DME Signal Shape}

Mathematically, a DME signal consists of pairs of Gaussian shaped pulses which are described by

$$
p(t)=e^{-\frac{\alpha}{2} t^{2}}+e^{-\frac{\alpha}{2}(t-\Delta t)^{2}} .
$$

The parameter $\Delta \mathrm{t}$ denotes the spacing of the pulses and depends on the certain mode of the DME station. The parameter $\alpha$ characterizes the width of one pulse. The used value $\alpha=4.5 \cdot 10^{11} \cdot 1 / \mathrm{s}^{2}$ leads to a width of $3.5 \mu \mathrm{s}$ at $50 \%$ of the maximum amplitude. Figure 4 clarifies the shape of one DME pulse pair and the given parameters.

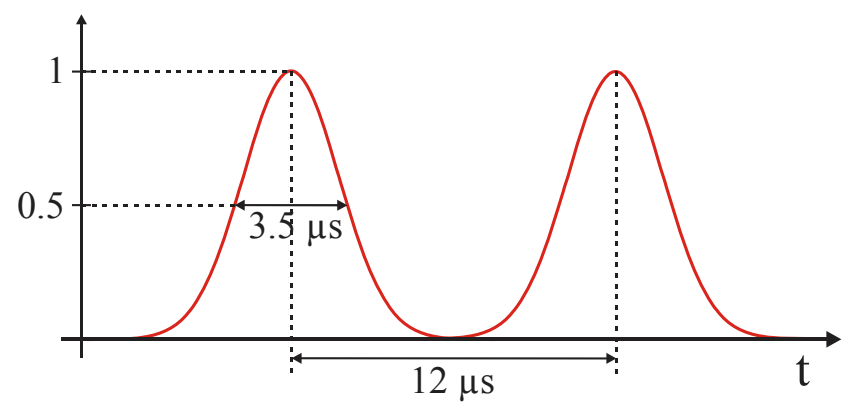

Figure 4. DME Pulse Pair in the Time Domain

A Gaussian shaped pulse also leads to a Gaussian shaped spectrum. However, since DME pulses always occur pair-wise the Gaussian shaped spectrum is modulated with a cosine. Mathematically this is described by

$$
I_{D M E}(f)=\sqrt{\frac{8 \pi}{\alpha}} e^{\frac{2 \pi^{2} t^{2}}{\alpha}} e^{-j \pi f \Delta t} \cos (\pi f \Delta t) .
$$


This baseband signal is modulated onto carrier frequencies in the aeronautical L-band from $960-1215 \mathrm{MHz}$ with a $1 \mathrm{MHz}$ channel grid. Figure 5 shows exemplarily a $4 \mathrm{MHz}$ detail of the L-band, comprising signals from four adjacent DME channels.

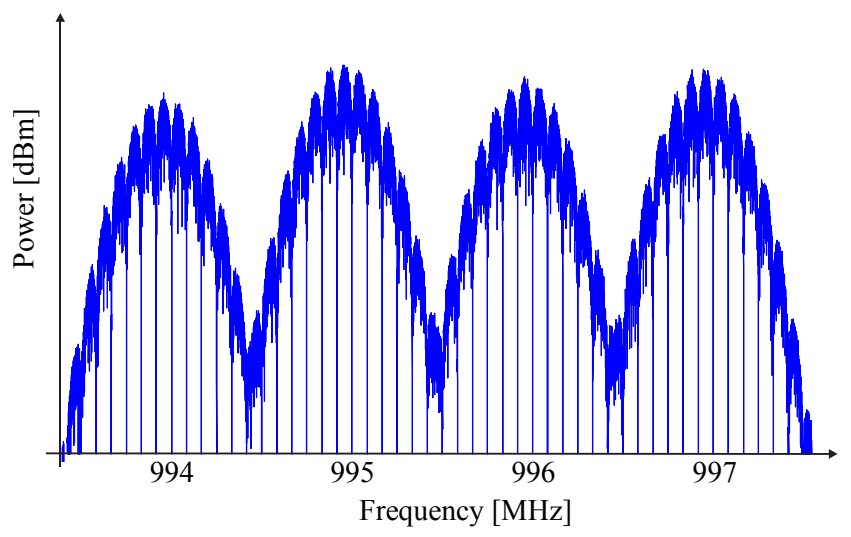

Figure 5. Spectrum of Four Adjacent DME Channels

\section{DME Principle}

The DME system is a transponder-based radio navigation technology that measures the slant range distance by measuring the propagation delay between a DME interrogator equipment onboard an aircraft and a transponder at a GS. Therefore, the DME interrogator at an AS sends a request pulse pair and the DME transponder replies after a fixed delay at a fixed frequency offset of $\pm 63 \mathrm{MHz}$, depending on the transmit frequency and the mode. Details on the assignment of the transponder frequency can be found in [6]. For X mode, the interrogator and the transponder transmit both with $\Delta \mathrm{t}=12 \mu \mathrm{s}$. In $\mathrm{Y}$ mode, the interrogator transmits with $\Delta \mathrm{t}=36 \mu \mathrm{s}$, while the transponder answers with $\Delta \mathrm{t}=30 \mu \mathrm{s}$. Note that the two other DME modes, $\mathrm{W}$ and $\mathrm{Z}$ mode are neglected in our case, since they are only used for $\mathrm{DME} / \mathrm{P}$ (precision). The DME/P is used together with the microwave landing system (MLS), which is employed only at very few airports in Europe.

A DME is usually coupled with a VHF omnidirectional range (VOR) device [7], which then allows the determination of the current position of the AS, when including information about the altitude. Alternatively the position can also be calculated based on trilateration if no VOR device is installed. Therefore one needs the estimated slant ranges between the AS interrogator and at least three GS transponders.

The transmission rate is given by the number of pulse pairs per second (ppps). A DME interrogator distinguishes between search and track mode. In search mode, it may transmit up to 150 ppps. When a connection to a transponder could be established, the rate decreases to 30 ppps. The number of replies sent by a DME ground transponder depends on the number of aircraft it has to serve. At maximum, 2700 ppps can be reached. However, DME ground stations automatically adjust their sensitivity level to maintain this constant squitter rate at all times. If the number of aircraft using a certain DME ground station would lead to a squitter rate less than 2700 ppps, the ground station reduces its sensitivity threshold. On the one hand, this allows aircraft farther away to be served by the DME as well. On the other hand, random fluctuations of the noise at the DME ground station receiver trigger spurious pulse transmissions by the DME ground station. Conversely, if the pulse rate is too high, the ground station increases its sensitivity threshold. Thus, the DME no longer responds to those aircraft that are farthest away. In this manner, the DME constantly adjusts its sensitivity in order to maintain a constant squitter rate of 2700 ppps. TACAN stations, which are used for military navigation, operate in a similar manner, but at a higher pulse rate of 3600 ppps.

\section{Interference Mitigation}

Since the power of DME pulses might be considerably above the LDACS1 power at an LDACS1 receiver, the DME interference significantly impairs the LDACS1 performance. For relieving this issue different interference mitigation approaches have been proposed [8]. In this investigation, we focus on pulse blanking $(\mathrm{PB})$, since it is easy to implement, while providing a remarkable performance gain. However, PB induces ICI, limiting the performance gain of PB. In [9], an algorithm for compensating the ICI has been proposed, which we also adopt in this paper.

\section{Pulse Blanking}

When applying PB all parts of the received signal, which exceed a certain pre-defined threshold, are set to zero. A critical issue when applying PB to an OFDM based system is the selection of the 
threshold $\mathrm{T}_{\mathrm{BN}}$. Since the OFDM signal itself has a strongly varying amplitude, it is difficult to distinguish between interference and signals peaks. Hence the threshold has to be selected as a trade-off between mitigating as much interference power as possible without losing too much useful signal. This issue has been addressed in [10].

In [11] the influence of the PB operation onto the OFDM signal and the AWGN has been investigated. A closed-form expression for the induced ICI has been derived, which turns out to affects all subcarrier evenly. An algorithm for removing the induced ICI, improving the system performance, is presented in the following.

\section{Pulse Blanking Compensation}

Pulse blanking compensation (PBC) is a scheme, which removes the ICI iteratively [9]. The ICI is completely described by the blanking positions, the transmitted modulation symbols, and the transmission channel coefficients. While the blanking positions are perfectly known at the receiver, the channel coefficients and the transmitted symbols are primarily not known. The idea of the algorithm is to obtain estimates for the channel coefficients and the transmitted symbols iteratively. Based on these estimates, it is possible to calculate estimates for the ICI and subtract them from the signal in the frequency domain. The block diagram, clarifying the functionality of the algorithm is depicted in Figure 1.

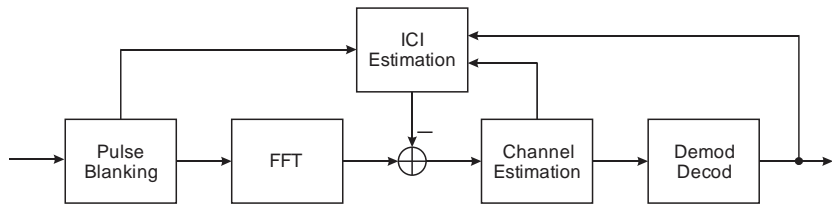

Figure 6. Block Diagram of ICI Compensation Principle

\section{Modeling of DME Impact onto LDACS1}

\section{Interference Map}

The LDACS1 FL in the frequency range $960-1009 \mathrm{MHz}$ is only affected by DME ground stations, as airborne DME interrogators are not active in this part of the spectrum. This greatly simplifies the characterization of the DME interference, since DME ground stations transmit at a practically constant pulse rate, as was described previously. Since the positions, channels, and transmit powers of the DME ground stations operating in Europe are known [12], it is possible to calculate, for any point in the airspace, which DME ground stations can be received at which power level, along with the expected pulse rate. This interference is completely independent of the amount of air traffic and the routes flown.

We have performed this analysis for the European airspace and for each of the 49 potential LDACS1 FL channels between $960.5 \mathrm{MHz}$ and $1008.5 \mathrm{MHz}$, in steps of $1 \mathrm{MHz}$. The geographic region between $35^{\circ} \mathrm{N}$ and $60^{\circ} \mathrm{N}$ and between $10^{\circ} \mathrm{W}$ and $30^{\circ} \mathrm{E}$ was divided into 2000 rectangular bins of one degree of longitude and 0.5 degrees of latitude. For each bin, we calculated the interference visible to an aircraft at an altitude of $10,000 \mathrm{~m}$ at the center of the bin. It is assumed that all ground stations within the aircraft's radio horizon are visible. An elevation model of the Earth's surface was not considered. Only the immediately adjacent DME channels are considered, i.e. those channels that are $500 \mathrm{kHz}$ above or below an LDACS channel. DME channels that are further away in the frequency domain are received at significantly lower power levels. The transmit power of the DME ground stations is given in [12] in terms of the equivalent isotropically radiated power (EIRP). From these values, the received power at the aircraft is calculated according to a free-space path loss as described by (3).

For each bin, we have determined the total number of pulse pairs received per second whose power is above $-80 \mathrm{dBm}$. It is assumed that pulses below this threshold contribute to the noise floor at the LDACS1 receiver. As an example, the interference map for the LDACS1 channel at 993.5 MHz is shown in Figure 7. This is a channel with strong interference. In particular is comprises the bin with the highest cumulative rate in Europe, which is 19,800 ppps in a bin near Dublin. Near London, the interference level is only slightly lower. For typical channels, the worst case interference is generally around 10,000 ppps, and channels with significantly lower interference can also be found for the European hotspot region around London, Paris, and Brussels. 


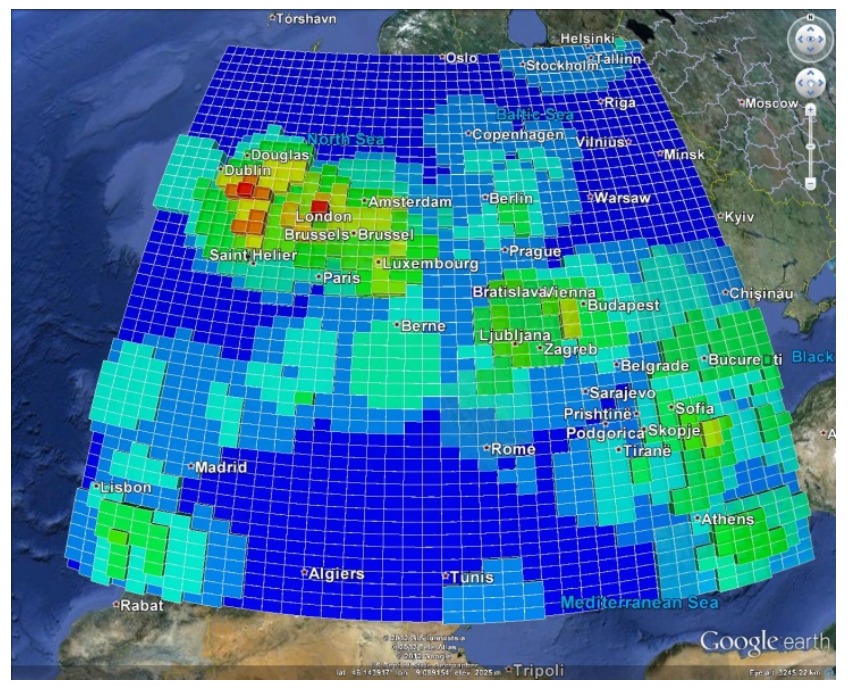

Figure 7. Interference map for LDACS channel 993.5 MHz

It should be noted that channels in the range 960.5 MHz-976.5 MHz are not assigned to DME or TACAN globally, but might be assigned on a national basis.

Our goal is to be able to use this interference map for purposes of frequency planning in a European LDACS1 network. That is, given a set of LDACS1 base station locations, allocate FL channels to these cells such that the DME interference in each cell is at a tolerable level. Of course, the representation of interference as a cumulative pulse rate as in Figure 7 is a simplification. To decide what is tolerable, we must define a model that allows the interference level in terms of the pulse rate, power distribution, and frequency offset of these pulses to be converted into the bit-error rate (BER) of the LDACS1 system that this interference leads to. This modeling process is addressed in the remainder of the paper.

Unfortunately, the situation in the LDACS1 RL is more complex. Here, only a small part of the DME interference is caused by DME ground stations. The largest part of the interference is due to the airborne DME interrogators. Therefore, the amount of interference depends on the amount of air traffic, as well as the actual DME stations that are being tracked by each aircraft. Due to this complex interaction, an analysis of the interference in the RL is not addressed in this paper. However, estimates of the future data traffic load, e.g. in the COCR [13], indicate that the data traffic on the FL will be much higher than on the
RL. Therefore, it is reasonable to focus on the FL, since this is the more critical of the two directions.

\section{Link Budget}

For determining the influence of the DME interference onto LDACS1, we have to derive both the LDACS1 power and the noise power at the airborne receiver. These values can be obtained by link budget calculations. An LDACS1 link budget is given in Table 2. In what follows, we explain the selected values. If not stated otherwise, the values are adopted from the link budget in [4].

Table 2. Parameters of LDACS1 Link Budget

\begin{tabular}{|l|c|c|c|}
\hline TX Parameters & Unit & Values & Values \\
\hline L-DACS1 TX ouput Power & $\mathrm{dBm}$ & 41 & 41 \\
\hline TX Antenna Gain & $\mathrm{dBi}$ & 6 & 8 \\
\hline Tx Cable loss (incl. Duplexer) & $\mathrm{dB}$ & 2 & 2 \\
\hline TX EiRP & $\mathrm{dBm}$ & 45 & 47 \\
\hline & & & \\
\hline Propagation Parameters & & & \\
\hline Transmit mid-band Frequency & $\mathrm{MHz}$ & 993.5 & 993.5 \\
\hline Tx-Rx Distance & $\mathrm{nm}$ & 120 & 120 \\
\hline Path Loss & $\mathrm{dB}$ & 139.33 & 139.33 \\
\hline & & & \\
\hline Miscellaneous Margins & & & \\
\hline Interference Margin & $\mathrm{dB}$ & 2 & 2 \\
\hline Implementation Margin & $\mathrm{dB}$ & 2 & 2 \\
\hline Safety Margin & $\mathrm{dB}$ & 6 & 6 \\
\hline Banking Loss Margin & $\mathrm{dB}$ & 0 & 0 \\
\hline & & & \\
\hline RX Parameters & & & \\
\hline Maximum RX Antenna Gain & $\mathrm{dBi}$ & 0 & 0 \\
\hline Rx Cable loss (incl. Duplexer) & $\mathrm{dB}$ & 3.5 & 3.5 \\
\hline L-DACS1 RX receive signal & $\mathrm{dBm}$ & -107.83 & -105.83 \\
\hline Thermal Noise Density $@ 290 \mathrm{~K}$ & $\mathrm{dBm} / \mathrm{Hz}$ & -174 & -174 \\
\hline Bandwidth & $\mathrm{Hz}$ & 498050 & 498050 \\
\hline Thermal Noise Power & $\mathrm{dBm}$ & -117.03 & -117.03 \\
\hline Receiver Noise Figure & $\mathrm{dB}$ & 6 & 6 \\
\hline Total Rx Noise Power & $\mathrm{dBm}$ & -111.03 & -111.03 \\
\hline & & & \\
\hline Resulting SNR & $\mathrm{dB}$ & 3.20 & 5.20 \\
\hline
\end{tabular}

\section{Transmitter Parameters}

The maximum LDACS1 GS transmit power is set to $41 \mathrm{dBm}$.

In [4] the antenna gain is given by $8 \mathrm{~dB}$. However, this gain depends on the chosen antenna and the elevation angle. For most DME antennas, which could also be adopted for LDACS1, the maximum gain is achieved for an elevation angle between $3^{\circ}$ and $5^{\circ}$ [14]. In this link budget, a transmitter-receiver distance of $120 \mathrm{NM}$ is assumed. In this case, a typical flight level would lead to an 
elevation angle around $1^{\circ}$, lowering the antenna gain up to $2 \mathrm{~dB}$ [14]. Taking this effect into account, we consider antenna gains between $6 \mathrm{~dB}$ and $8 \mathrm{~dB}$.

In an LDACS1 GS, cable losses of $2 \mathrm{~dB}$ are assumed. Due to separate transmit and receive antennas at the GS, no duplexer is needed.

These values add up to the EIRP.

\section{Transmission Channel Parameters}

The transmitter-receiver distance of $120 \mathrm{~nm}$ corresponds to the maximum planned cell radius for LDACS1. For larger radii an aircraft flying at an altitude below $4,000 \mathrm{~m}$ would vanish behind the radio horizon.

Assuming a line-of-sight transmission without reflections, the free-space path loss can be calculated according to

$$
L_{\text {free-space }}=\left(\frac{4 \pi d_{\mathrm{tx}-\mathrm{rx}} f_{c}}{c}\right)^{2} .
$$

The interference margin of $2 \mathrm{~dB}$ accounts for imperfections of the DME interference shape. In particular, measurement data indicates a more flat descent of the DME spectra, compared to the perfect shape, which is used for simulations. The system implementation margin is $2 \mathrm{~dB}$ below the value, given in [4], since in our simulations we applied a realistic channel estimation, which leads to roughly $2 \mathrm{~dB}$ performance degradation. A safety margin of $6 \mathrm{~dB}$ is obligatory for link budget considerations. For the considered en-route (ENR) case, a banking loss of $0 \mathrm{~dB}$ can be assumed.

These margins can be incorporated into the path loss, i.e., the attenuation of the LDACS1 signal.

\section{Receiver Parameters}

In an AS receiver, the cable losses are assumed to be larger than in the GS. In addition, a duplexer with a loss of $0.5 \mathrm{~dB}$ is assumed, leading to a total loss of $3.5 \mathrm{~dB}$.

Given the LDACS1 transmit power, the freespace path loss, margins, and the receiver cable and duplexer loss, we are now able to calculate the estimated received power of the LDACS1 signal.

The receiver noise power depends on the thermal noise power and the noise figure. A typical value for the noise figure of the components of the receiver signal chain is $6 \mathrm{~dB}$. The thermal noise power is calculated by multiplying the thermal noise density with the bandwidth of the useful LDACS1 signal.

Now, the SNR between the received LDACS1 signal and the receiver noise can be calculated and one obtains values between $3.2 \mathrm{~dB}$ and $5.2 \mathrm{~dB}$, according to the link budget from Table 2 .

\section{Derivation of Model for Equivalent SNR Loss}

One possibility for investigating the influence of the DME interference onto the performance of LDACS 1 is to carry out Monte Carlo simulations. However, when keeping the number of 2000 bins for all 49 possible DME FL channels in mind, the computational effort would be enormous. Thus, in this section we derive a model, which enables us to convert DME interference powers and pulse rates into an SNR loss of the transmission. This estimated SNR loss enables us to predict the system performance without carrying out extensive simulations.

In a first step, the received signal is filtered by a raised-cosine filter to reduce out-of-band radiation prior to the analog-digital conversion. Since the DME spectra have a $500 \mathrm{kHz}$ offset, remarkable parts of the interference are filtered out, leading to a peak attenuation of $27 \mathrm{~dB}$. Note that this value might be different when choosing another receive filter.

This is followed by the blanking nonlinearity. To determine whether DME pulses from a certain DME GS are blanked, the DME signal amplitudes after receiver filtering have to be compared to the blanking threshold $\mathrm{T}_{\mathrm{BN}}$. This threshold has to be scaled with the average LDACS1 received signal amplitude $^{1}$. Based on this comparison, the DME stations can be separated into stations, whose signals are blanked and stations, whose signals are not blanked.

For DME pulses below the threshold, i.e., nonblanked pulses from DME stations with index $k$, the interference power of one pulse can be calculated according to

\footnotetext{
${ }^{1}$ Note that the LDACS1 signal is not attenuated by the receiver filter, since the signal is completely within the pass band of the filter.
} 


$$
P_{\text {pulse }, k}=P_{\text {peak }, k} \int_{-\infty}^{+\infty} e^{-\frac{\alpha}{2} t^{2}} d t .
$$

Since pulses from a DME station $k$ always occur paired and with a fixed pulse rate $Q_{k}$, the average DME power of that station is calculated by

$$
P_{\mathrm{av}, k}=2 \cdot Q_{k} \cdot P_{\text {pulse }, k} .
$$

For DME pulses with index $l$ which are blanked, i.e., with signal parts above the threshold, we have to calculate the remaining interference power of signal parts below the threshold $T_{\mathrm{BN}}$. This is clarified in Figure 8.

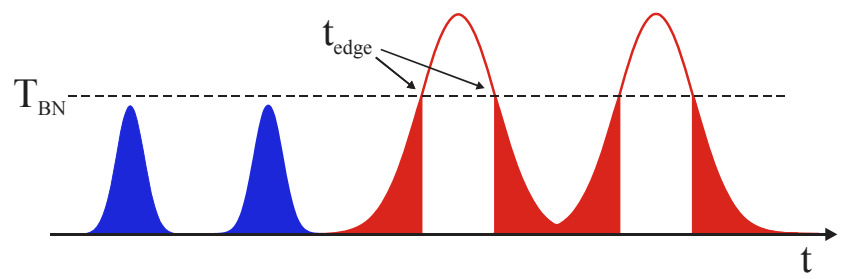

Figure 8. Remaining DME Parts after Pulse Blanking

First, the edges of the blanking window have to be calculated

$$
\begin{aligned}
& T_{\mathrm{BN}} \cdot \sqrt{E_{\mathrm{sig}}}=\sqrt{P_{\text {peak }, l}} \cdot e^{-\frac{\alpha}{2} t_{\text {edge }, l}^{2}} \\
& t_{\text {edge } 1 / 2, l}= \pm \sqrt{-\frac{2}{\alpha} \ln \left(\frac{T_{\mathrm{BN}} \cdot \sqrt{E_{\text {sig }}}}{\sqrt{P_{\text {peak }, l}}}\right)} .
\end{aligned}
$$

Based on these edge positions, we are now able to calculate the remaining interference power, given by

$$
P_{\text {rem }, l}=P_{\text {peak }, l}\left(\int_{-\infty}^{t_{\text {edge }, l}} e^{-\frac{\alpha}{2} t^{2}} d t+\int_{t_{\text {edge }, l}}^{+\infty} e^{-\frac{\alpha}{2} t^{2}} d t\right) .
$$

According to (5), the total inference power of DME stations which are blanked is calculated by

$$
P_{\mathrm{av}, l}=2 \cdot Q_{l} \cdot P_{\mathrm{rem}, l} \text {. }
$$

Next, we are interested in which way the interference power calculated in (5) and (8) spreads over the LDACS1 subcarriers. Due to the guard bands at the edges of the transmission bandwidth, the DME power affects the LDACS1 signal in the frequency domain only partly. Simulations showed that roughly half of the DME power is outside the used subcarriers. In addition, since DME pulses comprise a center frequency offset of $\pm 500 \mathrm{kHz}$, they affect either only the left or the right edge of the LDACS1 transmission bandwidth. Hence we split this bandwidth into three parts each comprising $N_{\text {part }}$ subcarriers and calculate a separate SNR for each part. The principle is clarified in Figure 9. The average interference power in the left part is obtained by summing up the interference power from all DME stations with a center frequency offset of $-500 \mathrm{kHz}$. For obtaining the average interference power in the right part the interference power from all DME stations with a center frequency offset of $+500 \mathrm{kHz}$ have to be summed up. The adding of powers is justified, since the signals from different DME stations are independent. This computes to

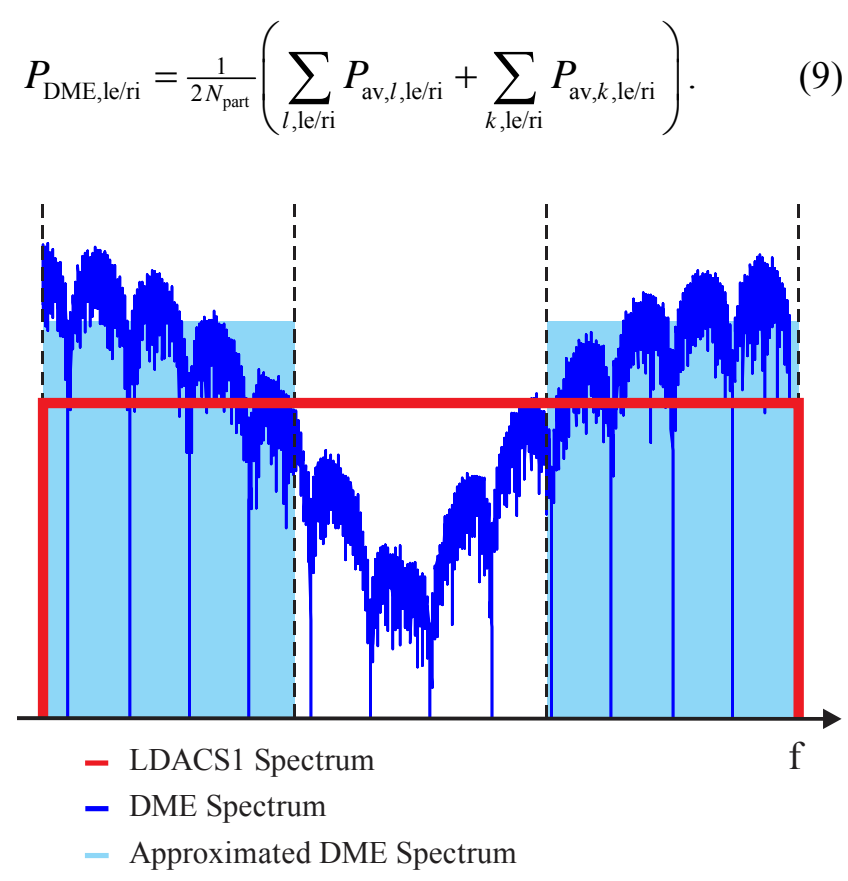

Figure 9. Approximation of DME Spectrum

Next we are interested in the average ratio $B_{l}$ of time, during which the received signal is blanked due to pulses from the lth DME station

$$
B_{l}=2 \cdot Q_{l} \cdot\left(t_{\text {edge } 2, l}-t_{\text {edge } 1, l}\right) .
$$

Since the DME pulses from the different stations occur independently, the joint average ratio for not blanking the received signal is calculated by

$$
K_{\mathrm{av}}=\prod_{l=1}^{L}\left(1-B_{l}\right)
$$

with $L$ being the total number of DME stations whose pulses are blanked. Note that the entire LDACS1 
bandwidth, i.e. all three parts, is affected in the same way by blanking in the time domain, which was shown in [11].

We are now able to calculate equivalent SNR values for the three parts of the LDACS1 spectrum separately. The required LDACS1 signal and AWGN power can be adopted from the link budget in Table 2. Since the middle part of the spectrum is only affected by the blanking, but not by the remaining interference, we obtain

$$
S N R_{\text {mid }}=\frac{K_{\mathrm{av}}^{2} \cdot E_{\mathrm{sig}}}{K_{\mathrm{av}} \cdot N_{0}+K_{\mathrm{av}}\left(1-K_{\mathrm{av}}\right) \cdot E_{\mathrm{sig}}} .
$$

This formula has been derived in [11]. The numerator comprises the attenuated power of the LDACS1 signal, $E_{\text {sig. }}$ The denominator consists of attenuated noise power $N_{0}$ and ICI, which is induced by the blanking.

For the left and right part of the spectrum, (12) has to be extended by the interference power and we obtain

$$
S N R_{\mathrm{le} / \mathrm{ri}}=\frac{K_{\mathrm{av}}^{2} E_{\mathrm{sig}}}{K_{\mathrm{av}} N_{0}+K_{\mathrm{av}}\left(1-K_{\mathrm{av}}\right) E_{\mathrm{sig}}+P_{\mathrm{DME}, \mathrm{le} / \mathrm{ri}}} .
$$

When the ICI is mitigated by PBC, (13) has to be adapted. In this case the ICI term is scaled by an ICI mitigation factor $\alpha_{\mathrm{ICI}}$. This factor depends on the BER after $\mathrm{PB}$, since the PBC is based on estimates of the transmitted bits. Former investigations [9] showed that a gain by $\mathrm{PBC}$ is observed for BER $<1 \cdot 10^{-1}$. For $\mathrm{BER}<1 \cdot 10^{-4}$, the ICI is almost perfectly estimated. Between these two BER values, simulations showed that a mapping by a quartic equation to calculate $\alpha_{\mathrm{ICI}}$ leads to the best results. This writes

$$
\alpha_{\mathrm{ICI}}=\left\{\begin{array}{cc}
0, & B E R<1 \cdot 10^{-4}, \\
1, & B E R>1 \cdot 10^{-1},(14) \\
\left(\frac{1}{3}\left(\log _{10}(\mathrm{BER})+4\right)\right)^{4}, & \text { else },
\end{array}\right.
$$

and we obtain for the SNR after PBC

$$
S N R_{\mathrm{le} / \mathrm{ri}}=\frac{K_{\mathrm{av}}^{2} E_{\mathrm{sig}}}{K_{\mathrm{av}} N_{0}+\alpha_{\mathrm{ICI}} K_{\mathrm{av}}\left(1-K_{\mathrm{av}}\right) E_{\mathrm{sig}}+P_{\mathrm{DME}, \mathrm{le} / \mathrm{ri}}} .
$$

Finally, an overall equivalent SNR is obtained by averaging over the three spectral parts

$$
S N R_{\mathrm{equ}}=\frac{S N R_{\mathrm{le}}+S N R_{\mathrm{mid}}+S N R_{\mathrm{ri}}}{3} .
$$

We are aware that this equivalent SNR is only an approximation, since the DME signal occurs impulsive but not constant, making an averaging questionable. However, there are some facts justifying our assumption:

- Although the interference occurs only for short periods of time, the FFT spreads the interference power over an entire OFDM symbol

- In the LDACS1 FL, the coding and especially the interleaver sizes are rather high, which leads to a spread of the interference within OFDM symbols, but also over multiple OFDM symbols.

In the next section we verify our algorithm for calculating an equivalent SNR, by comparing the results to simulations when applying realistic interference.

\section{Simulation Results}

After having derived a model for the equivalent SNR of a LDACS1 transmission exposed to DME interference we now confirm the model based on simulations. Additionally, this model is applied to identify critical bins and channel assignments, where the LDACS1 performance suffers most.

\section{Confirmation of Model}

For comparing the model derived in the previous section to realistic simulation results, we first have to obtain BER values by simulations for an undistorted transmission. For the simulations, we selected the most robust ACM parameter set with a CC rate of 0.5 , RS coding with rate 0.9 , and QPSK modulation. We applied a realistic ENR channel model. For estimating the channel transfer function in the receiver, two-dimensional Wiener filtering is employed. The BER results are shown in Figure 10 for $\mathrm{CC}$ only and the concatenation of $\mathrm{CC}$ and $\mathrm{RS}$ coding vs. the SNR. This enables us either to transform the equivalent SNR into a BER, or simulated BER values into corresponding SNR values. 


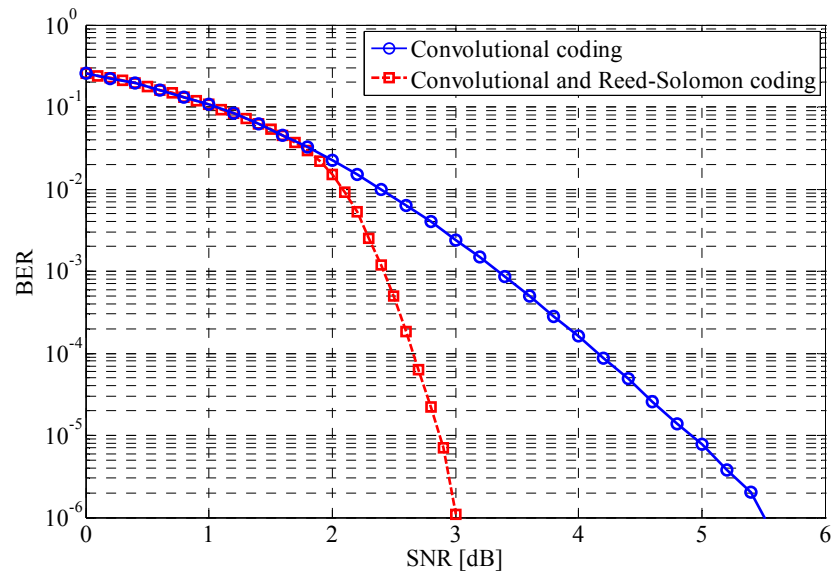

Figure 10. BER of LDACS1, without Interference

For verifying our model, we selected the LDACS1 transmit frequency of $993.5 \mathrm{MHz}$, which was already identified above as a channel with strong DME interference.

In a first step we calculate equivalent SNR values for $\mathrm{PB}$ and $\mathrm{PBC}$ according to (16). Next we select the corresponding BER values from Figure 10. These values are compared to simulated BER values. The results are compared in Figure 11 for $\mathrm{PB}$ and $\mathrm{CC}$ only, in Figure 12 for $\mathrm{PB}$ with $\mathrm{CC}$ and RS coding, and in Figure 13 for $\mathrm{PBC}$ with $\mathrm{CC}$ and $\mathrm{RS}$ for $\mathrm{SNR}=3.2 \mathrm{~dB}$. The figures indicate the good match of the derived model. They also visualize the beneficial influence of the concatenated coding scheme and the iterative ICI compensation.
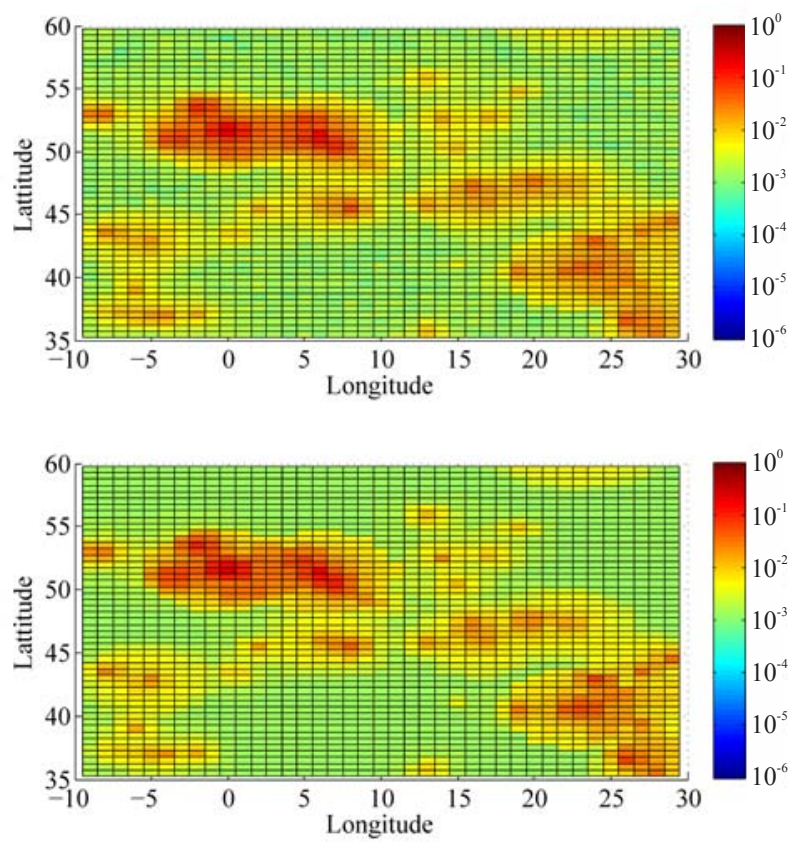

Figure 11. Simulated (top) and Estimated (bottom) BER after CC, with PB, SNR = 3.2 dB.
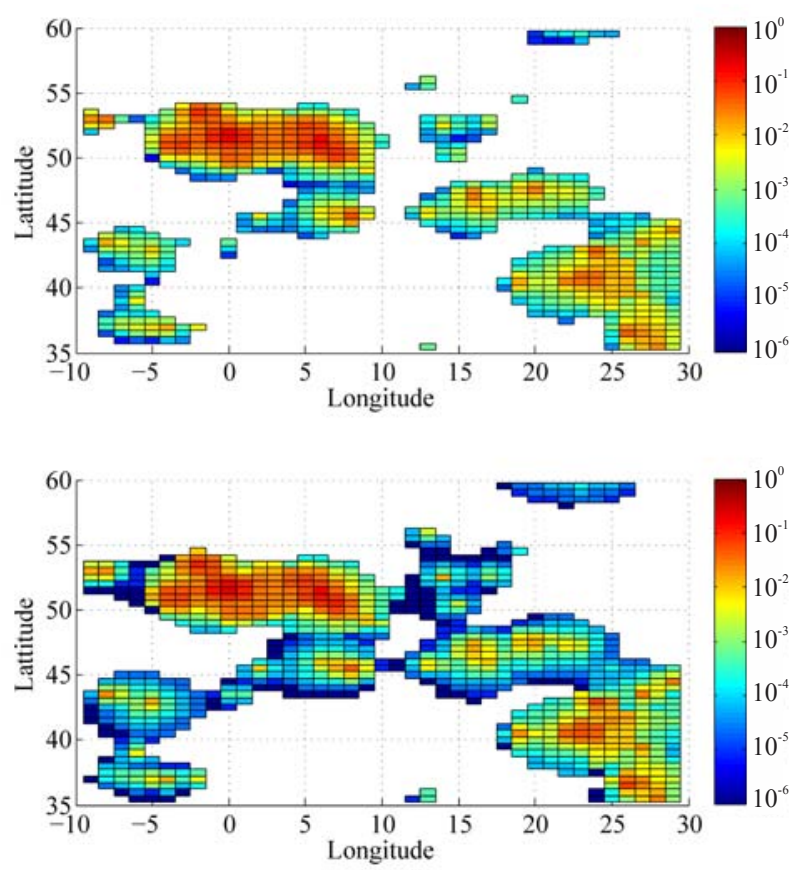

Figure 12. Simulated (top) and Estimated (bottom) BER after CC and RS Coding, with PB, $\mathrm{SNR}=3.2 \mathrm{~dB}$. 

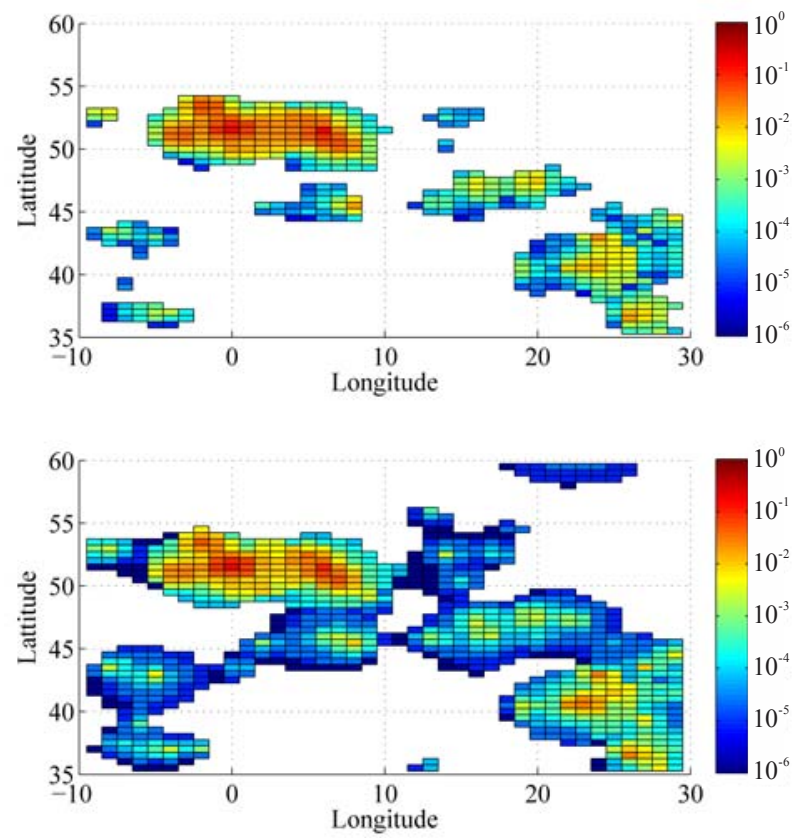

Figure 13. Simulated (top) and Estimated (bottom) BER after CC and RS Coding, with $\mathrm{PBC}, \mathrm{SNR}=3.2 \mathrm{~dB}$.

For applying our model to different LDACS1 frequencies and SNR values, we have to confirm the model matching not only qualitatively but also quantitatively. Therefore the average relative error $\varepsilon$ of the BER is given in Table 3 for different values of SNR. We compare BER values obtained by simulation with BER values, adopted from Figure 10. Averaging covers all bins of the interference map. It turns out that the derived model fits the simulated results well independently of the chosen SNR. However, the model degrades from $\mathrm{CC}$ to $\mathrm{CC}$ with RS coding. Since the BER curve for CC with RS coding is steeper than the CC curve in Figure 10, a mismatch in the calculation of the equivalent SNR has a more severe influence onto the BER mismatch. For PBC, an additional degradation of the model can be observed. Obviously, the fraction of compensated ICI can never be perfectly estimated, which introduces an additional error source. However, the relative error in the BER estimation is still below 2, which is acceptable when estimating the BER. The estimated error for $\mathrm{PBC}$ with $\mathrm{SNR}=4.7 \mathrm{~dB}$ is not very reliable, since the number of bins, in which any bit errors could be observed is very small. For $\mathrm{SNR}=5.2 \mathrm{~dB}$, no bit errors at all could be observed when applying PBC.
Table 3. Relative Error of BER Estimation

\begin{tabular}{|c|c|c|c|}
\hline $\mathbf{S N R}$ & $\boldsymbol{\varepsilon}\left(\mathbf{B E R}_{\mathbf{C C}+\mathbf{P B}}\right)$ & $\boldsymbol{E}_{\left(\mathbf{B E R}_{\mathbf{R S}+\mathbf{P B}}\right)}$ & $\boldsymbol{E}_{\left(\mathbf{B E R}_{\mathbf{R S}+\mathbf{P B C}}\right)}$ \\
\hline 3.2 & 0.16 & 0.73 & 1.41 \\
\hline 3.7 & 0.26 & 0.83 & 1.57 \\
\hline 4.2 & 0.40 & 0.83 & 1.12 \\
\hline 4.7 & 0.36 & 0.79 & 4.80 \\
\hline 5.2 & 0.30 & 0.19 & -- \\
\hline
\end{tabular}

Another measure for assessing the quality of the model is the error of the SNR estimation itself. Therefore, we transform the simulated BER into an SNR value according to Figure 10 and compare it to the calculated equivalent SNR from (16). The results are presented in Table 4. In this case, we are not interested in the relative error. Since the SNR is given in the logarithmic dB-scale, we calculated the average absolute error $\varepsilon$ of the SNR estimation. Interestingly, the performance of the SNR estimation does neither depend on the chosen coding and interference mitigation, nor on the SNR in a significant way. Only for $\mathrm{SNR}=5.2 \mathrm{~dB}$ a slight degradation is observed. However, as mentioned above, the number of bins with BER values above zero is very low in this case, impairing the significance of the results. In any other case, the average SNR estimation error is below $0.2 \mathrm{~dB}$.

Table 4. Absolute Error of SNR Estimation

\begin{tabular}{|c|c|c|c|}
\hline SNR & $\boldsymbol{\varepsilon}_{\left(\mathbf{S N R}_{\mathbf{C C}+\mathbf{P B}}\right)}$ & $\boldsymbol{\varepsilon}_{\left(\mathbf{S N R}_{\mathbf{R S}+\mathbf{P B}}\right)}$ & $\boldsymbol{\varepsilon}_{\left(\mathbf{S N R}_{\mathbf{R S}+\mathbf{P B C}}\right)}$ \\
\hline 3.2 & 0.07 & 0.07 & 0.09 \\
\hline 3.7 & 0.09 & 0.08 & 0.11 \\
\hline 4.2 & 0.13 & 0.11 & 0.11 \\
\hline 4.7 & 0.14 & 0.11 & 0.20 \\
\hline 5.2 & 0.60 & 0.77 & -- \\
\hline
\end{tabular}

In summary, it can be stated that our proposed algorithm performs sufficiently well and we apply it in the following for assessing the interference impact for different LDACS1 channels.

\section{Performance Evaluation}

After having confirmed the validity of our derived model, we are now able to assess the expected performance of all possible LDACS1 frequencies based on this model.

In [4], BER $<1 \cdot 10^{-6}$ has been defined as performance criterion for a successful LDACS1 
transmission. In the following we derive the number of LDACS1 channels failing this criterion, in dependence of the bin. This is carried out for several SNR values between $3.2 \mathrm{~dB}$ and $5.2 \mathrm{~dB}$.

For identifying critical bins, we created a map, indicating the number of critical LDACS1 channels, i.e., channels with a BER $>1 \cdot 10^{-6}$. It is depicted in Figure 14 for $\mathrm{SNR}=3.7 \mathrm{~dB}$. The red colored bins have 13 critical channels. The most challenging areas for successfully operating LDACS1 are around London, Brussels, and Amsterdam. Note, there are still non-critical channels available in these areas. Interestingly, the area around Athens is also worth of considering, while the interference situation around hotspots like Paris, Rome or Madrid, is less severe.

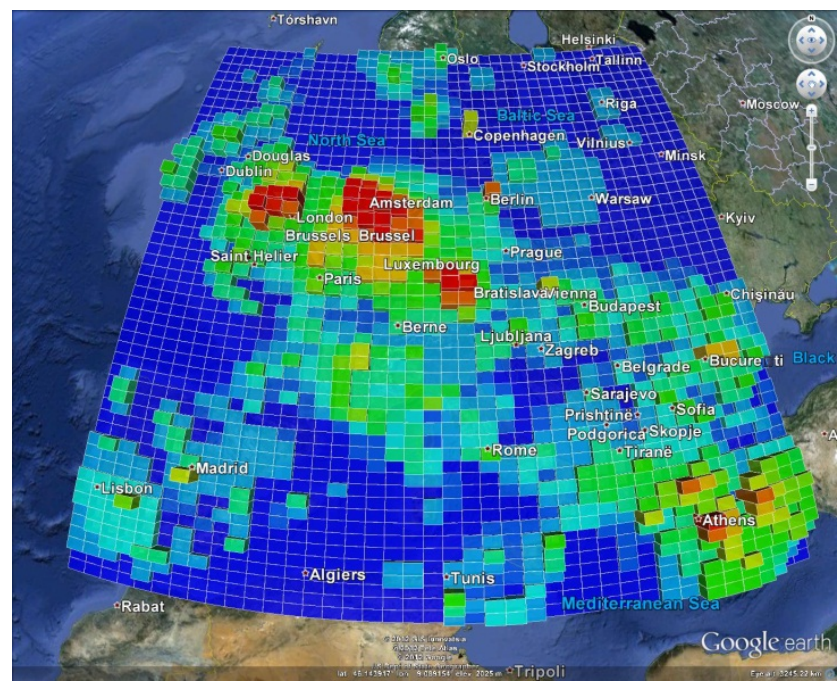

Figure 14. Map of Critical LDACS1 Channels for $\mathrm{SNR}=3.7 \mathrm{~dB}$

Finally we investigate the dependence of the number of critical bins on the SNR. In Figure 15, the cumulative number of critical bins is plotted vs. the number of critical LDACS1 channels for several SNR values. The difference between two adjacent values of a curve indicates the number of bins, which fail the BER criterion for the respective number of LDACS1 channels $\mathrm{N}_{\text {crit. }}$. The total number represents the number of bins, which fail the criterion for at least one LDACS1 channel, but at most $\mathrm{N}_{\text {crit. }}$ This is of particular interest when assessing the number of bins with at least one critical LDACS1 channel, since in this case frequency planning has to be applied. It turns out that the number of critical bins depends quite strongly on the SNR. If the SNR is high, e.g.
$5.2 \mathrm{~dB}$, only a very small fraction of LDACS1 channels have critical bins. In this case, frequency planning for the LDACS1 network would be necessary, but this would be a simple task On the other hand, if the SNR is only $3.2 \mathrm{~dB}$, practically all of the 2000 bins have at least one critical channel. In this case, frequency planning may turn out to be challenging, since a non-critical channel must be found for each bin. Note that one LDACS1 cell would cover multiple bins of the interference map. Additional constraints also need to be considered, such as minimum separation between cells using the same channel. This topic will be addressed in future work.

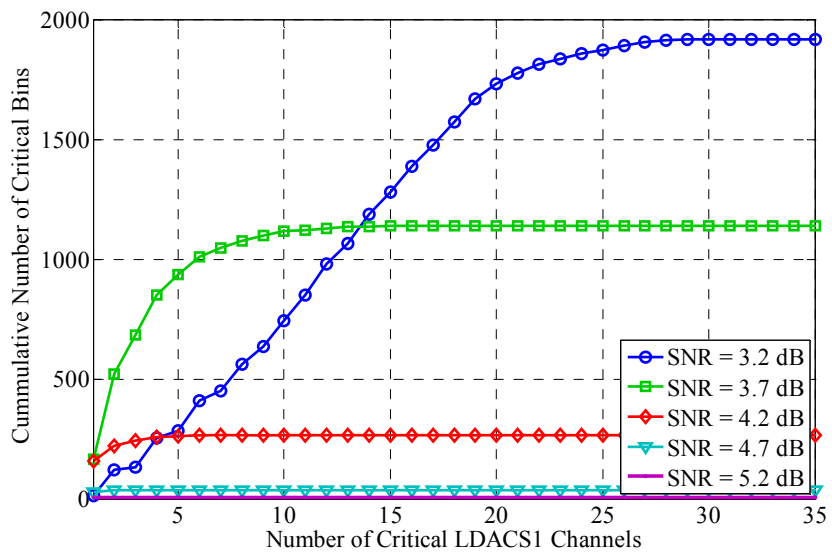

Figure 15. Cumulative Number of Critical Bins vs. Number of Critical LDACS1 Channels for Several SNR Values

\section{Conclusions}

In this paper, we derive a model for characterizing the DME impact on LDACS1, without carrying out extensive simulations. The idea is to transform the DME power and pulse rate into an equivalent degradation of the performance of an interference-free LDACS1 transmission. Simulations show a good accordance with realistic simulations. Based on this model, we assess the interference situation for the DME interference in Europe. It turns out that LDACS1 can cope with DME interference; however frequency planning is needed under certain conditions. This is subject to further research. In addition, the investigation could easily be extended to different continents, given only information about the parameters of the DME stations. 


\section{References}

[1] "http://www.sesarju.eu/."

[2] "http://www.faa.gov/nextgen/."

[3] M. Sajatovic, B. Haindl, M. Ehammer, T. Gräupl, M. Schnell, U. Epple, and S. Brandes, "LDACS1 System Definition Proposal: Deliverable D2, Edition 1.0, Eurocontrol Study."

[4] "https://www.eurocontrol.int/communications/public/standard_page/LDACS1_Update.html.”

[5] U. Epple and M. Schnell, "Overview of Interference Situation and Mitigation Techniques for LDACS1," in Proc. Digital Avionics System Conference, Oct. 2011.

[6] ICAO, "International Standards and Recommended Practices, Aeronautical Telecommunications, Annex 10 to the Convention on International Civil Aviation, Volume I (Radio Navigation Aids)," July 2006.

[7] M. Kayton and W. Fried, Avionics Navigation Systems. Wiley, 2nd edition, 1997.

[8] M. Schnell, S. Brandes, S. Gligorevic, M. Walter, C. Rihacek, M. Sajatovic, and B. Haindl, "Interference Mitigation for Broadband L-DACS," in 27th Digital Avionics Systems Conference (DASC), St. Paul, MN, USA, October 2008.

[9] S. Brandes, U. Epple, and M. Schnell, "Compensation of the Impact of Interference Mitigation by Pulse Blanking in OFDM Systems," in Proc. IEEE Globecom Telecomm. Conf., Nov. 2009.
[10] S. Brandes and M. Schnell, "Interference Mitigation for the Future Aeronautical L-Band Communication System," in 7th Intl. Workshop on Multi-Carrier Systems \& Solutions (MC-SS 2009), Herrsching, Germany, S. P. et al., Ed., May 2009.

[11] U. Epple, K. Shibli, and M. Schnell, "Investigation of Blanking Nonlinearity in OFDM Systems," in IEEE Int.Comm. Conf., Kyoto, Japan, June 2011.

[12] "http://www.paris.icao.int/documents_open/show_file.php?id=279."

[13] EUROCONTROL \& FAA, "Communications Operating Concept and Requirements for the Future Radio System, (COCR)," 2007.

[14] EUROCONTROL, "B-AMC Deliverable D4, Interference Analysis and Spectrum Requirements," 2007.

\section{Email Addresses}

Ulrich.Epple@,DLR.de

Felix.Hoffmann@DLR.de

Michael.Schnell@,DLR.de

2012 Integrated Communications Navigation and Surveillance (ICNS) Conference

April 24-26, 2012 